\title{
Parameter Sizing for Fluid Power Circuits Using Taguchi Methods
}

\author{
A.M.Connor \\ Engineering Design Centre, Department of Engineering, University of Cambridge, Trumpington Street, \\ Cambridge, CB2 1PZ, United Kingdom.
}

\begin{abstract}
This paper describes the application of Taguchi methods [1,2,3] to the parameter sizing stage of fluid power system design. Taguchi methods have become almost synonymous with robust design and are used to design systems that are tolerant to the effects of noise factors. Noise factors are defined as anything that causes changes in the functional characteristics or performance of the system that are not controllable. In the hydraulic circuit example used in this paper, these noise factors are assumed to be effects of component failure. The method is therefore being used to select design parameter values such that the resulting circuits exhibit some tolerance to the initial development of faults in the system which will allow the system to continue to operate for a short period of time without catastrophic failure occurring.
\end{abstract}

\section{Introduction}

The robust design approach consists of three distinct phases. These phases are conceptual design, parameter design and tolerance design. In the conceptual design phase a number of different solution concepts are generated that are functionally capable of solving the engineering problem being considered. This phase of the design process often utilises such tools as Quality Function Deployment (QFD) and other aspects of systematic design to ensure that as large a number of suitable concepts are generated.

During the parameter design phase the specific controllable factors in the chosen concept that effect the robustness are identified as well as the uncontrollable noise factors. The optimum values for the control factors are chosen so that the system performance is as consistent as possible for a range of different noise factor scenarios. Finally, during the tolerance design phase the allowable control factor variability is determined and allowable limits for additional noise factors are calculated.

In this paper, Taguchi methods are used to optimise the parameters of a hydraulic circuit so that it is tolerant to the initial development of faults in the system components. Whilst failures such as a broken vane in a pump can be detected by monitoring system pressure and output power, a fault tolerant system may allow a brief period of continued operation in order to allow maintenance to be scheduled to coincide with other planned downtime without secondary failures occurring.

In the example presented in this paper it is assumed that the design concept used is suitable for the task and no additional noises will be present. Therefore only the parameter design phase is considered. In many respects this can be viewed as an alternative approach to the use of numerical optimisation techniques to aid in the sizing and selection of fluid power components $[4,5,6]$.

\section{Taguchi Methods}

There are several important concepts that need to be explored in any description of Taguchi methods. Most of these concepts are adequately covered in the existing literature $[1,2,3]$ but will briefly described here. Taguchi methods have been applied with varying degrees of 
success to a wide range of problems including manufacturing control [7] and structural optimisation $[8,9]$.

\subsection{Robustness and Quality}

The perception of quality in an artefact is closely related to the sensitivity of the design to noise factors. Noise factors can be defined as potential inputs to the system which cannot be controlled. Good examples include environmental factors such as temperature variation. In order to achieve a high quality design it is necessary to eliminate any variability in performance. There are two courses of action which may be followed. The first of these is to eliminate the actual source of noise but this can be costly, time consuming and ineffective if the factors are too complex to control easily. The second option is to eliminate the artefacts sensitivity to the source of noise. The artefact can then be said to be robust even though the sources of noise have not been eliminated.

This robustness can be related to quality through two fundamental factors [10] that can be used to assess the quality of an artefact. These are the features of the artefact and conformance to those features. The performance of a design solution to meet a need can be viewed as a feature. The ability for that design solution to meet the need through a wide range of conditions can be viewed as conformance, so providing a direct link between robustness and quality.

\subsection{Signal to Noise Ratios}

Given that there is a direct relationship between quality and robustness it is important to represent the performance of a design across all of the noise conditions for which the design is intended to be insensitive. The signal to noise ratio $(\mathrm{S} / \mathrm{N})$ metrics are intended for use in the optimisation of an artefacts robust performance.

A number of different $\mathrm{S} / \mathrm{N}$ ratios can be used depending on the aims of the optimisation process. However, all $\mathrm{S} / \mathrm{N}$ ratios have the following characteristics [2].

- The $\mathrm{S} / \mathrm{N}$ ratio reflects the variability in the response of the system caused by the noise factors.

- The $\mathrm{S} / \mathrm{N}$ ratio is independent of the adjustment of the mean. This implies that the metric would be useful for predicting quality even if the target value should change.

- The $\mathrm{S} / \mathrm{N}$ ratio measures relative quality because it is to be used for comparative purposes.

- The S/N ratio does not induce unnecessary complications, such as control factor interactions, when the influences of many factors on product quality are analysed.

One example of the different $\mathrm{S} / \mathrm{N}$ ratios is the smaller-the-better type that is used in this paper. This $\mathrm{S} / \mathrm{N}$ ratio is generally used when the response values of the system under consideration are continuous and nonnegative and the desired response value is zero. A practical example would be the minimisation of a speed error between the actual speed of a motor and a desired speed.

Other $\mathrm{S} / \mathrm{N}$ ratios include the larger-the-better type, the operating window type and the nominal-the-best type. These are not used in this paper but are used when different performance requirements dominate the design. 
The smaller-the-better $\mathrm{S} / \mathrm{N}$ ratio is calculated by using equation 1 .

$$
\frac{S}{N}=-10 \log \left[\frac{1}{n} \sum_{i=1}^{n} y_{i}^{2}\right]
$$

In this equation the performance metric $y$ of a design solution is found for $n$ different noise conditions which then allows the $\mathrm{S} / \mathrm{N}$ ratio to be calculated.

One key aspect of the robust design approach is the decision for which design parameter combinations the $\mathrm{S} / \mathrm{N}$ ratio is calculated and this is usually achieved through the use of orthogonal arrays.

\subsection{Orthogonal Arrays}

A number of different approaches can be used in the design of experiments. The orthogonal array approach is a method of setting up experiments that only require a fraction of the full factorial combinations but yet explores a significant proportion of the total possible solution space. This possibly provides the most efficient approach.

A typical orthogonal array is shown below in Table 1.

Table 1. L8 Orthogonal Array

\begin{tabular}{cccccccc}
\hline Run & A & B & C & D & E & F & G \\
\hline $\mathbf{1}$ & 1 & 1 & 1 & 1 & 1 & 1 & 1 \\
$\mathbf{2}$ & 1 & 1 & 1 & 2 & 2 & 2 & 2 \\
$\mathbf{3}$ & 1 & 2 & 2 & 1 & 1 & 2 & 2 \\
$\mathbf{4}$ & 1 & 2 & 2 & 2 & 2 & 1 & 1 \\
$\mathbf{5}$ & 2 & 1 & 2 & 1 & 2 & 1 & 2 \\
$\mathbf{6}$ & 2 & 1 & 2 & 2 & 1 & 2 & 1 \\
$\mathbf{7}$ & 2 & 2 & 1 & 1 & 2 & 2 & 1 \\
$\mathbf{8}$ & 2 & 2 & 1 & 2 & 1 & 1 & 2 \\
\hline
\end{tabular}

This is a two level standard L8 orthogonal array. There are seven possible design parameters, each of which may take one of two possible values. Eight experiments are carried out where each of the parameters has the value indicated by a 1 or a 2 .

The art to developing good orthogonal array experiments is choosing the most appropriate design parameters and assigning them to the correct columns. Not all columns need be filled and a poor assignment may lead to problems with interactions.

Typically, an array such as the L8 array will be used to determine the parameter value combinations that will be searched. However, there are two possible approaches for introducing noise into the experiments. The first option is to carry out an independent noise experiment where the effects of a number of noise factors and noise factor levels are considered for a nominal set of design parameter values. The noise factor effects can then be lumped together into two compound noise factors which represent best and worst case. This approach has several drawbacks in that the individual noise factor effects become hidden and it is necessary to have some idea of nominal operating values for the design 
parameters. For these reasons, the approach used in this paper is the second option which utilises crossed array experiments. The typical structure of a crossed array experiment is shown in Table 2.

Table 2. Crossed Array Experiment

\begin{tabular}{|c|c|c|c|c|c|c|c|c|c|c|c|c|c|c|c|}
\hline Run & $\overline{\mathbf{A}}$ & B & $\bar{C}$ & D & $\mathbf{E}$ & $\mathbf{F}$ & $\mathbf{G}$ & $\mathbf{H}$ & & & & & & Mean & S/N Ratio \\
\hline & & & & & & & & & & 1 & 2 & 3 & 4 & & \\
\hline & & & & & & & & & $\mathrm{R}$ & $\mathrm{L}$ & $\mathrm{H}$ & $\mathrm{H}$ & $\mathrm{L}$ & & \\
\hline & & & & & & & & & Q & $\mathrm{L}$ & $\mathrm{H}$ & $\mathrm{L}$ & $\mathrm{H}$ & & \\
\hline & & & & & & & & & $\mathrm{P}$ & $\mathrm{L}$ & $\mathrm{L}$ & $\mathrm{H}$ & $\mathrm{H}$ & & \\
\hline 1 & 1 & 1 & 1 & 1 & 1 & 1 & 1 & 1 & & $\mathrm{y}_{1}$ & $\mathrm{y}_{2}$ & $\mathrm{y}_{3}$ & $\mathrm{y}_{4}$ & $(\Sigma \mathrm{y} / 4)_{1}$ & $\mathrm{~S} / \mathrm{N}_{1}$ \\
\hline 2 & 1 & 1 & 2 & 2 & 2 & 2 & 2 & 2 & & $\mathrm{y}_{1}$ & $\mathrm{y}_{2}$ & $\mathrm{y}_{3}$ & $\mathrm{y}_{4}$ & $(\Sigma \mathrm{y} / 4)_{2}$ & $\mathrm{~S} / \mathrm{N}_{2}$ \\
\hline 3 & 1 & 1 & 3 & 3 & 3 & 3 & 3 & 3 & & $\mathrm{y}_{1}$ & $\mathrm{y}_{2}$ & $\mathrm{y}_{3}$ & $\mathrm{y}_{4}$ & $(\Sigma \mathrm{y} / 4)_{3}$ & $\mathrm{~S} / \mathrm{N}_{3}$ \\
\hline 4 & 1 & 2 & 1 & 1 & 2 & 2 & 3 & 3 & & $\mathrm{y}_{1}$ & $\mathrm{y}_{2}$ & $\mathrm{y}_{3}$ & $\mathrm{y}_{4}$ & $(\Sigma \mathrm{y} / 4)_{4}$ & $\mathrm{~S} / \mathrm{N}_{4}$ \\
\hline 5 & 1 & 2 & 2 & 2 & 3 & 3 & 1 & 1 & & $\mathrm{y}_{1}$ & $\mathrm{y}_{2}$ & $\mathrm{y}_{3}$ & $\mathrm{y}_{4}$ & $(\Sigma \mathrm{y} / 4)_{5}$ & $\mathrm{~S} / \mathrm{N}_{5}$ \\
\hline 6 & 1 & 2 & 3 & 3 & 1 & 1 & 2 & 2 & & $\mathrm{y}_{1}$ & $\mathrm{y}_{2}$ & $\mathrm{y}_{3}$ & $\mathrm{y}_{4}$ & $(\Sigma y / 4)_{6}$ & $\mathrm{~S} / \mathrm{N}_{6}$ \\
\hline 7 & 1 & 3 & 1 & 2 & 1 & 3 & 2 & 3 & & $\mathrm{y}_{1}$ & $\mathrm{y}_{2}$ & $\mathrm{y}_{3}$ & $\mathrm{y}_{4}$ & $(\Sigma \mathrm{y} / 4)_{7}$ & $\mathrm{~S} / \mathrm{N}_{7}$ \\
\hline 8 & 1 & 3 & 2 & 3 & 2 & 1 & 3 & 1 & & $\mathrm{y}_{1}$ & $\mathrm{y}_{2}$ & $\mathrm{y}_{3}$ & $\mathrm{y}_{4}$ & $(\Sigma \mathrm{y} / 4)_{8}$ & $\mathrm{~S} / \mathrm{N}_{8}$ \\
\hline 9 & 1 & 3 & 3 & 1 & 3 & 2 & 1 & 2 & & $\mathrm{y}_{1}$ & $\mathrm{y}_{2}$ & $\mathrm{y}_{3}$ & $\mathrm{y}_{4}$ & $(\Sigma \mathrm{y} / 4)_{9}$ & $\mathrm{~S} / \mathrm{N}_{9}$ \\
\hline 10 & 2 & 1 & 1 & 3 & 3 & 2 & 2 & 1 & & $\mathrm{y}_{1}$ & $\mathrm{y}_{2}$ & $\mathrm{y}_{3}$ & $\mathrm{y}_{4}$ & $(\Sigma \mathrm{y} / 4)_{10}$ & $\mathrm{~S} / \mathrm{N}_{10}$ \\
\hline 11 & 2 & 1 & 2 & 1 & 1 & 3 & 3 & 2 & & $\mathrm{y}_{1}$ & $\mathrm{y}_{2}$ & $\mathrm{y}_{3}$ & $\mathrm{y}_{4}$ & $(\Sigma \mathrm{y} / 4)_{11}$ & $\mathrm{~S} / \mathrm{N}_{11}$ \\
\hline 12 & 2 & 1 & 3 & 2 & 2 & 1 & 1 & 3 & & $\mathrm{y}_{1}$ & $\mathrm{y}_{2}$ & $\mathrm{y}_{3}$ & $\mathrm{y}_{4}$ & $(\Sigma \mathrm{y} / 4)_{12}$ & $\mathrm{~S} / \mathrm{N}_{12}$ \\
\hline 13 & 2 & 2 & 1 & 2 & 3 & 1 & 3 & 2 & & $\mathrm{y}_{1}$ & $\mathrm{y}_{2}$ & $\mathrm{y}_{3}$ & $\mathrm{y}_{4}$ & $(\Sigma y / 4)_{13}$ & $\mathrm{~S} / \mathrm{N}_{13}$ \\
\hline 14 & 2 & 2 & 2 & 3 & 1 & 2 & 1 & 3 & & $\mathrm{y}_{1}$ & $\mathrm{y}_{2}$ & $\mathrm{y}_{3}$ & $\mathrm{y}_{4}$ & $(\Sigma \mathrm{y} / 4)_{14}$ & $\mathrm{~S} / \mathrm{N}_{14}$ \\
\hline 15 & 2 & 2 & 3 & 1 & 2 & 3 & 2 & 1 & & $\mathrm{y}_{1}$ & $\mathrm{y}_{2}$ & $\mathrm{y}_{3}$ & $\mathrm{y}_{4}$ & $(\Sigma y / 4)_{15}$ & $\mathrm{~S} / \mathrm{N}_{15}$ \\
\hline 16 & 2 & 3 & 1 & 3 & 2 & 3 & 1 & 2 & & $\mathrm{y}_{1}$ & $\mathrm{y}_{2}$ & $\mathrm{y}_{3}$ & $\mathrm{y}_{4}$ & $(\Sigma y / 4)_{16}$ & $\mathrm{~S} / \mathrm{N}_{16}$ \\
\hline 17 & 2 & 3 & 2 & 1 & 3 & 1 & 2 & 3 & & $\mathrm{y}_{1}$ & $\mathrm{y}_{2}$ & $\mathrm{y}_{3}$ & $\mathrm{y}_{4}$ & $(\Sigma y / 4)_{17}$ & $\mathrm{~S} / \mathrm{N}_{17}$ \\
\hline 18 & 2 & 3 & 3 & 2 & 1 & 2 & 3 & 1 & & $\mathrm{y}_{1}$ & $\mathrm{y}_{2}$ & $\mathrm{y}_{3}$ & $\mathrm{y}_{4}$ & $(\Sigma \mathrm{y} / 4)_{18}$ & $\mathrm{~S} / \mathrm{N}_{18}$ \\
\hline
\end{tabular}

In this experiment a three level L18 array is used for the inner array of design parameters and a smaller two level L4 array is used for the noise factors. For each design parameter combination the response is determined for each noise factor combination. This allow both the mean response and the signal to noise ratio to be calculated.

In general, the solution that exhibits the best performance has the highest signal to noise ratio. In many published applications of Taguchi methods the best solution from the orthogonal array is selected and claimed to be the optimal solution. However, this simple selection does not exhibit the power of the method and by using an analysis of means (ANOM) it is possible to determine the contribution of each parameter on the robustness of the performance and hence select the best levels for each parameter. It is likely that the proposed solution will have a parameter combination that does not appear in the orthogonal array but exhibits a more robust performance.

\subsection{Analysis of Means}

The analysis of means provides an approach to determine the contribution of each design parameter, or factor, on the overall robustness of the solution. The analysis of means is done by taking average values for the performance metric that correspond with the factor levels. Consider the simple example shown in Table 3 which shows an L4 arrays used in with a single noise state. 
Table 3. L4 Orthogonal Array

\begin{tabular}{ccccc}
\hline Run & A & B & C & \\
\hline $\mathbf{1}$ & 1 & 1 & 1 & $\mathrm{y}_{1}$ \\
$\mathbf{2}$ & 1 & 2 & 2 & $\mathrm{y}_{2}$ \\
$\mathbf{3}$ & 2 & 1 & 2 & $\mathrm{y}_{3}$ \\
$\mathbf{4}$ & 2 & 2 & 1 & $\mathrm{y}_{4}$ \\
\hline
\end{tabular}

For example, the factor effects for factor B at each level can be calculated using equation 2 and equation 3.

$$
\begin{aligned}
& \bar{y}_{B 1}=\left(y_{1}+y_{3}\right) / 2 \\
& \bar{y}_{B 2}=\left(y_{2}+y_{4}\right) / 2
\end{aligned}
$$

Plotting these values on a simple graph would show something similar to that in Figure 1 which has plotted factor effects in $\mathrm{S} / \mathrm{N}$ ratios.
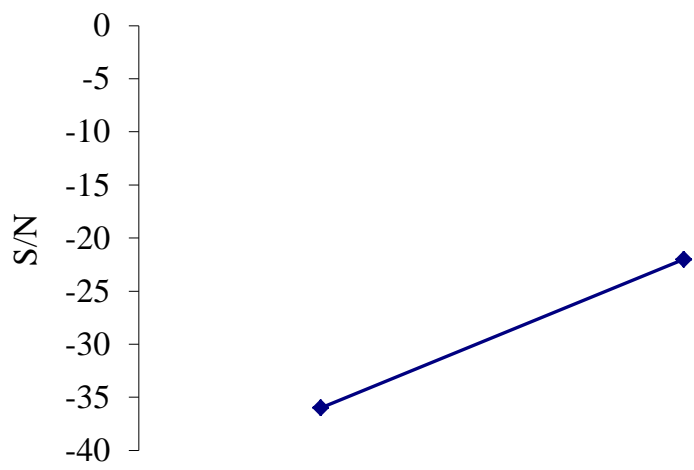

$\mathrm{B}(1)$

$\mathrm{B}(2)$

Figure 1. Simple Factor Effect Plot

By comparing the range of the factor effect for each factor or design parameter it is possible to determine which factors have the greatest effect on system robustness. It is also possible to determine potentiality optimal parameter settings by selecting the level for each parameter that exhibits the highest average $\mathrm{S} / \mathrm{N}$ levels. In the above example, the parameter B should be set to level 2 .

\section{Fluid Power System Design Example}

The circuit considered in this example is shown in Figure 2. The functional aim of the circuit is to ramp the load up to a constant speed of $300 \mathrm{rpm}$ and maintain that speed despite there being a step change of applied load torque after two seconds. This functionality is achieved through the use of a PI controller and a proportional servo valve. This circuit has been considered in previous work [5] where the circuit parameters were optimised using a Tabu search algorithm. In this previous work a smaller number of control factors were used and the circuit was only optimised for a single operating condition and therefore the effects of noise were not considered. 


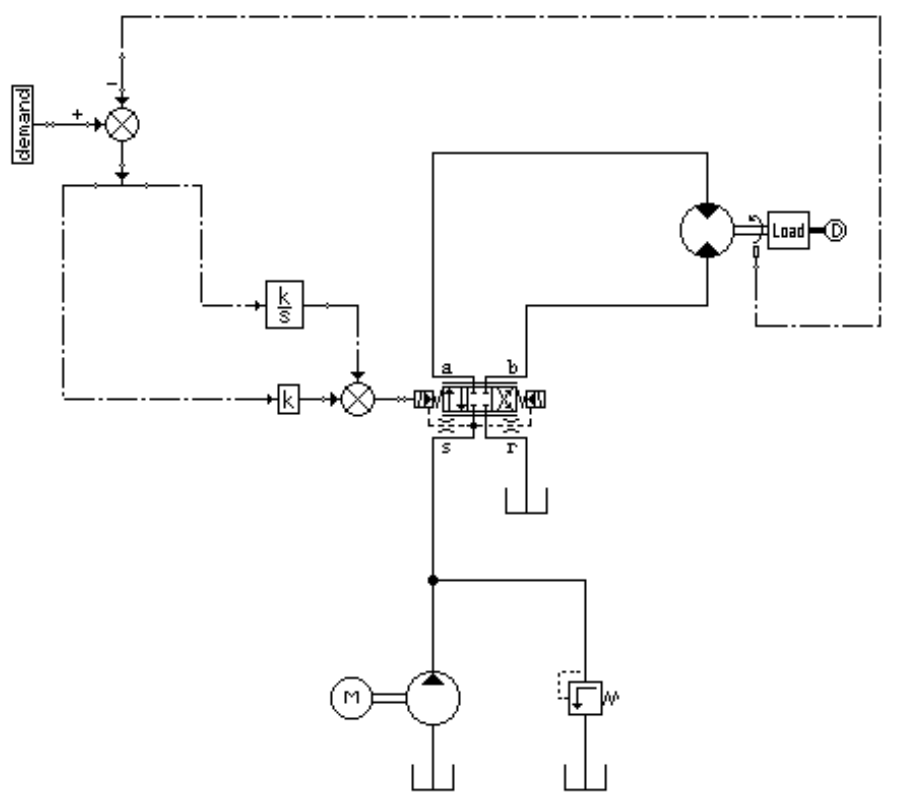

Figure 2. Hydraulic test circuit

\subsection{Selection of Noise Factors and Control Factors}

An important aspect of the parameter design phase is the selection of both the noise factors and the control factors to be considered. It is essential that the noise factors used represent the actual variabilities that are likely to cause dissatisfactions. Noise factors are defined as factors that can cause fluctuations in the performance of a system but are not controllable in normal use. The noise factors selected in this example are intended to represent failed components. The noise factors used are the slip loss of both the pump and motor, null leakage in the servo valve and the friction of the load.

Increased slip losses are intended to represent a faulty pump or motor which may have failed in such a way as a broken vane or piston which results in less fluid being supplied to the circuit. Null leakage in the servo valve is being used to consider a spool valve that has become significantly underlapped which then results in a leakage between ports without losses occurring out of the system. Finally, an increased load friction is being used to represent a faulty bearing. Two terms are used which represent the stiction torque friction and the steady state coulomb friction.

The selection of control factors is also of vital importance. The selected control factors must have the ability to introduce robustness into the design by minimising the effects due to noise. The control factors used in this work are the gains for the PI controller, the pump displacement, the motor displacement and the servo valve current rating.

Noise factors and control factors are normally illustrated through the use of a P-diagram which shows that both types influence the system performance. The P-diagram for this example is shown in Figure 3. 


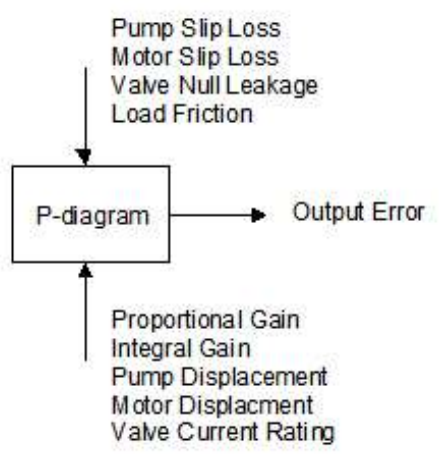

Figure 3. P-Diagram

Given the noise and control factors it is now possible to define a quality performance metric and then design experiments using crossed orthogonal arrays as described in section 2.3 .

\subsection{Quality Metric}

The performance of each solution for each simulation is determined by applying equation 3.1. In this equation the squared error between the desired speed and actual speed at each time step in the simulation is further penalised by considering the proportion of the pump flow rate that is returning to tank through the relief valve.

$$
\text { obfn }=\sum_{i=0}^{n}\left[\left(\omega_{\text {desired }}-\omega_{\text {actual }}\right)^{2} \times\left(1+\frac{Q_{r v}}{Q_{p}}\right)\right]
$$

This extra penalty term is included to force the method towards finding solutions that exhibit good operating characteristics as well as being robust to the effects of noise.

\subsection{Parameter Optimisation Experiments}

A crossed array experiment has been used to analyse the response of different parameter values and noise conditions. Crossed array experiments were discussed in section 2.3.

In the experiment the outer array of noise factors is a standard L9 array that allows for three levels for four different noise factors. This is shown in Table 4.

Table 4. Outer Array of Noise Factors

\begin{tabular}{ccccc}
\hline Run & $\begin{array}{c}\text { Pump } \\
\text { Slip Loss }\end{array}$ & $\begin{array}{c}\text { Motor } \\
\text { Slip Loss }\end{array}$ & $\begin{array}{c}\text { Valve } \\
\text { Null Leakage }\end{array}$ & $\begin{array}{c}\text { Load } \\
\text { Friction }\end{array}$ \\
\hline $\mathbf{1}$ & Low & Low & Low & Low \\
$\mathbf{2}$ & Low & Medium & Medium & Medium \\
$\mathbf{3}$ & Low & High & High & High \\
$\mathbf{4}$ & Medium & Low & Medium & High \\
$\mathbf{5}$ & Medium & Medium & High & Low \\
$\mathbf{6}$ & Medium & High & Low & Medium \\
$\mathbf{7}$ & High & Low & High & Medium \\
$\mathbf{8}$ & High & Medium & Low & High \\
$\mathbf{9}$ & High & High & Medium & Low \\
\hline
\end{tabular}


The three levels for each noise factor each correspond to real values as shown in Table 5.

Table 5. Noise Factor Level

\begin{tabular}{cccc}
\hline Factor & High & Medium & Low \\
\hline $\begin{array}{c}\text { Pump slip-loss } \\
\text { coefficient } \\
\text { Motor slip-loss } \\
\text { coefficient }\end{array}$ & $3 \times 10^{-8}$ & $1.65 \times 10^{-8}$ & $3 \times 10^{-9}$ \\
$\begin{array}{c}\text { Valve null leakage } \\
\text { Load friction }\end{array}$ & $3 \times 10^{-8}$ & $1.65 \times 10^{-8}$ & $3 \times 10^{-9}$ \\
(stiction torque) & $0.75 \mathrm{l} / \mathrm{min}$ per $150 \mathrm{Bar}$ & $0.50 \mathrm{l} / \mathrm{min}$ per $150 \mathrm{Bar}$ & 0 \\
$\begin{array}{c}\text { Load friction } \\
\text { coloumb friction) }\end{array}$ & $50 \mathrm{Nm}$ & $30 \mathrm{Nm}$ & 0 \\
\hline
\end{tabular}

A modified L16 array is used for the control factors in the inner array which is shown in Table 6. This array has been modified so as to provide four different levels for five parameters. The array has been modified in this way so as to provide a greater number of levels for each control factor. One criticism of Taguchi methods when compared to numerical optimisation approaches is the restricted number of levels each control factor can take. In the crossed array approach, each parameter value combination is run for each of the noise factor combinations in the outer array. The response and performance metric is calculated for each control factor combination for each noise condition, though only the mean error and $\mathrm{S} / \mathrm{N}$ ratio is shown for each control factor combination in Table 6 . The signal to noise ratio used is the 'smaller-the-better' type as shown in equation 1.

Table 6. Inner Array of Control Factors and Response

\begin{tabular}{cccccccc}
\hline Run & $\mathbf{K}_{\mathbf{P}}$ & $\mathbf{K}_{\mathbf{I}}$ & $\begin{array}{c}\text { Pump } \\
\text { Displacement }\end{array}$ & $\begin{array}{c}\text { Motor } \\
\text { Rating }\end{array}$ & $\begin{array}{c}\text { Valve } \\
\text { Rating }\end{array}$ & $\begin{array}{c}\text { Mean } \\
\text { Response }\end{array}$ & $\begin{array}{c}\text { S/N } \\
\text { Ratio }\end{array}$ \\
\hline $\mathbf{1}$ & 0.25 & 10 & 60 & 300 & 150 & 34078.24 & -92.7667 \\
$\mathbf{2}$ & 0.25 & 20 & 80 & 400 & 200 & 23640.76 & -89.4589 \\
$\mathbf{3}$ & 0.25 & 30 & 100 & 500 & 250 & 19501.82 & -87.728 \\
$\mathbf{4}$ & 0.25 & 40 & 120 & 600 & 300 & 17925.28 & -86.8557 \\
$\mathbf{5}$ & 0.5 & 10 & 80 & 300 & 300 & 4748.614 & -73.565 \\
$\mathbf{6}$ & 0.5 & 20 & 60 & 400 & 250 & 492092.2 & -113.963 \\
$\mathbf{7}$ & 0.5 & 30 & 120 & 600 & 200 & 16975.53 & -86.4175 \\
$\mathbf{8}$ & 0.5 & 40 & 100 & 500 & 150 & 116248.2 & -101.394 \\
$\mathbf{9}$ & 0.75 & 10 & 100 & 600 & 200 & 243329.7 & -107.864 \\
$\mathbf{1 0}$ & 0.75 & 20 & 120 & 500 & 150 & 1850.204 & -66.8796 \\
$\mathbf{1 1}$ & 0.75 & 30 & 60 & 400 & 300 & 470021.1 & -113.579 \\
$\mathbf{1 2}$ & 0.75 & 40 & 80 & 300 & 250 & 1599214 & -124.089 \\
$\mathbf{1 3}$ & 1.0 & 10 & 120 & 400 & 250 & 3668.911 & -71.3196 \\
$\mathbf{1 4}$ & 1.0 & 20 & 100 & 300 & 300 & 3104.06 & -70.9323 \\
$\mathbf{1 5}$ & 1.0 & 30 & 80 & 600 & 150 & 770003.4 & -117.766 \\
$\mathbf{1 6}$ & 1.0 & 40 & 60 & 500 & 200 & 978676.7 & -120.224 \\
\hline
\end{tabular}

The best solution in the orthogonal array is found in experiment 10 . This solution has the lowest mean error and therefore the highest $\mathrm{S} / \mathrm{N}$ ratio value. The actual response of this solution can be considered on a number of noise factor conditions. The three conditions considered are when all the noise factors are set to low, medium and high. By considering 
the noise factor combinations in Table 4 it can be seen that only one of these noise conditions has been used in the parameter optimisation experiment.

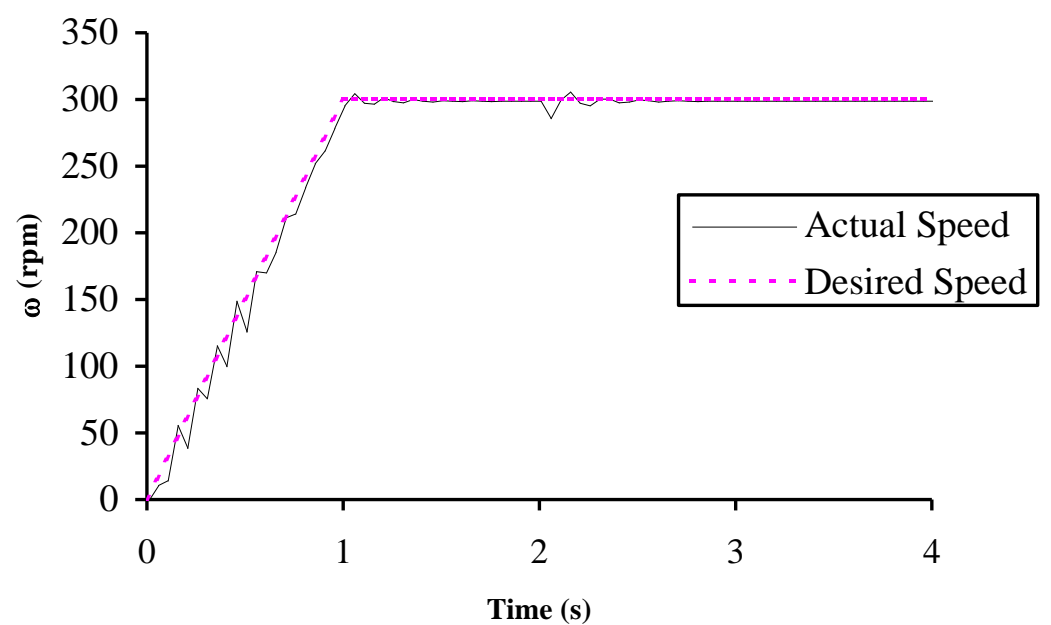

Figure 4. Response of best solution for noise factors at low values

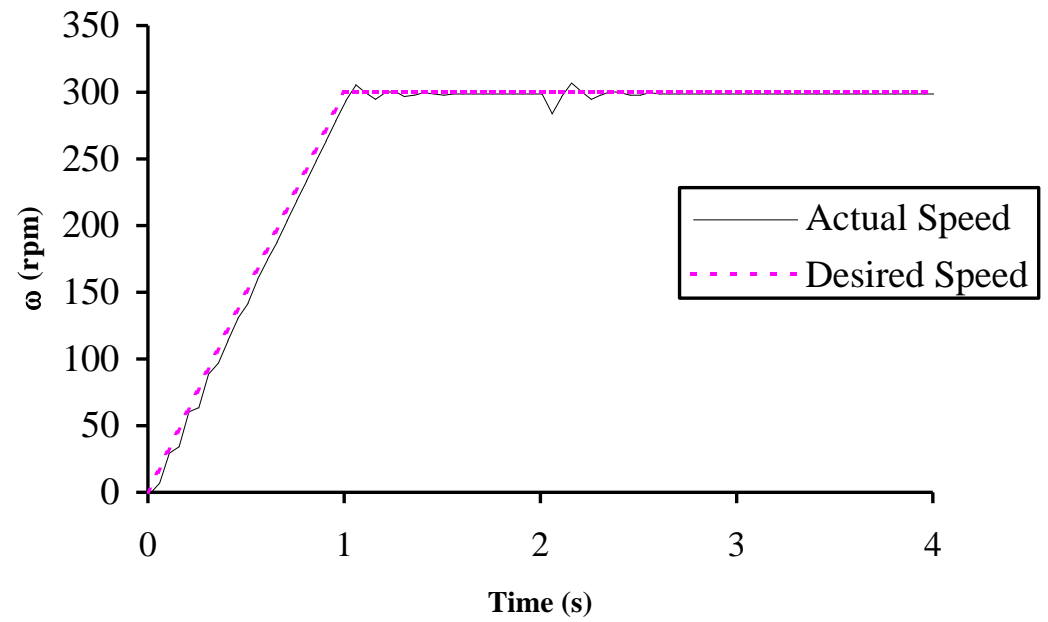

Figure 5. Response of best solution for noise factors at medium values 


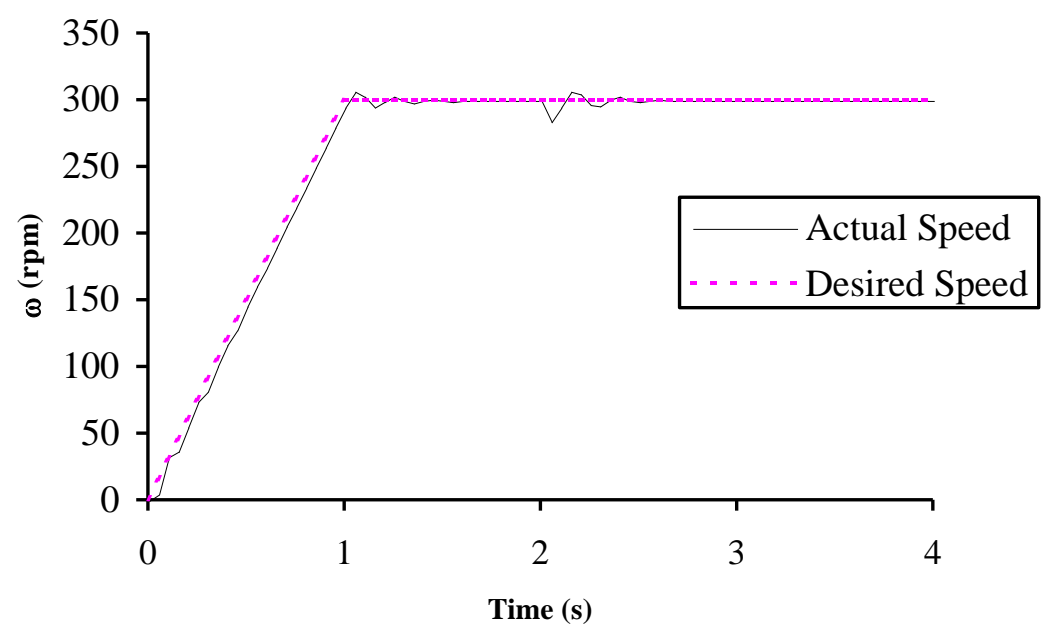

Figure 6. Response of best solution for noise factors at high values

From these results it is possible to determine the effects on response of each of the control factors using an Analysis of Means (ANOM) approach. The effects on the response for each factor at each level are given below in tabular form.

Table 7. Results of ANOM

\begin{tabular}{cccc}
\hline Factor & Level & Error (Average) & Error $\mathbf{( S / N )}$ \\
\hline $\mathbf{K}_{\mathbf{P}}$ & 0.25 & 23786.53 & -89.20 \\
& 0.50 & 157516.14 & -93.84 \\
& 0.75 & 578603.80 & -103.10 \\
$\mathbf{K}_{\mathbf{I}}$ & 1.00 & 438863.26 & -95.06 \\
& 10 & 71456.37 & -86.38 \\
& 20 & 130171.81 & -85.31 \\
Pump Displacement & 30 & 319125.45 & -101.37 \\
& 40 & 678016.09 & -108.14 \\
Motor Displacement & 60 & 493717.04 & -110.13 \\
& 80 & 599401.75 & -101.22 \\
& 100 & 95545.95 & -91.98 \\
& 120 & 10104.98 & -77.87 \\
Valve Rating & 400 & 413580.44 & -93.66 \\
& 500 & 247355.74 & -97.08 \\
& 600 & 262059.22 & -94.06 \\
& 150 & 230545.01 & -99.73 \\
& 200 & 315655.67 & -94.70 \\
& 250 & 528619.29 & -100.99 \\
& 300 & 123949.76 & -86.27 \\
\hline
\end{tabular}

This information can also be plotted graphically to assess the significance of each factor. Figure 7 shows the $\mathrm{S} / \mathrm{N}$ values plotted for each level of each factor. 


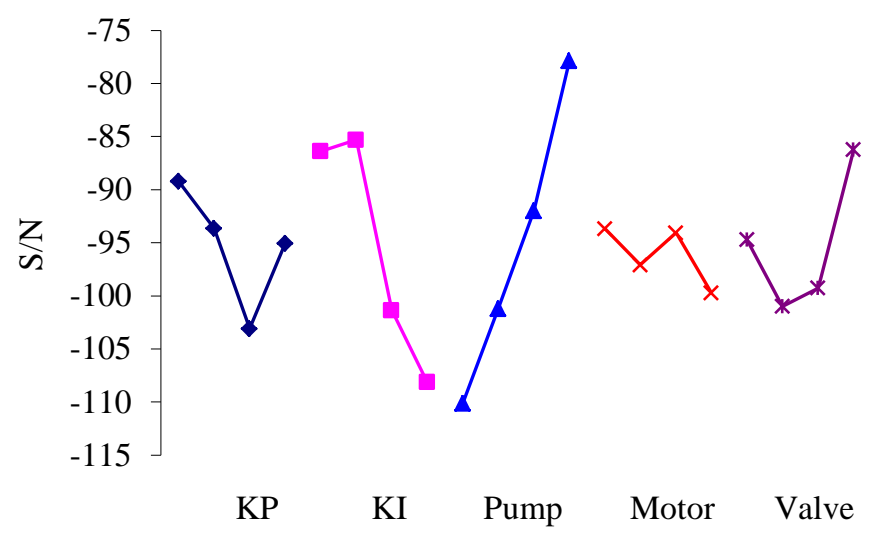

Figure 7. Factor effect plots

It can be seen that each factor has a different effect on the robustness of the system. An optimal parameter set can be proposed by considering the relationship between signal to noise ratio and system performance. In general, lowest speed error is likely to occur for the parameters chosen so that the $\mathrm{S} / \mathrm{N}$ ratio is maximised for each factor. The proposed optimal parameter set is therefore

$$
\begin{array}{ll}
\mathrm{K}_{\mathrm{P}}=0.25 & \mathrm{M}=300 \mathrm{cc} / \mathrm{rev} \\
\mathrm{K}_{\mathrm{I}}=20 & \mathrm{~V}=300 \mathrm{~mA} \\
\mathrm{P}=120 \mathrm{cc} / \mathrm{rev} &
\end{array}
$$

However, the range of variation for the motor displacement is very small and it is recommended that this be used as a 'fine tuning' control parameter. The output performance for each of the possible solutions can now be considered. The response figure of merit has been calculated for three noise conditions, low medium and high for each potential solution.

Table 8. Performance values for noise factors set to low, medium and high values

\begin{tabular}{cccc}
\hline $\begin{array}{c}\text { Motor } \\
\text { Displacement }\end{array}$ & $\begin{array}{c}\text { Response of low } \\
\text { noise }\end{array}$ & $\begin{array}{c}\text { Response for medium } \\
\text { noise }\end{array}$ & $\begin{array}{c}\text { Response for high } \\
\text { noise }\end{array}$ \\
\hline $\mathbf{3 0 0}$ & 31830.28 & 12440.16 & 15594.59 \\
$\mathbf{4 0 0}$ & 3716.20 & 4045.21 & 4925.62 \\
$\mathbf{5 0 0}$ & 2960.74 & 3334.91 & 3777.80 \\
$\mathbf{6 0 0}$ & 3208.69 & 17764.12 & 40867.85 \\
\hline
\end{tabular}

It can be seen that the predicted optimal solution in fact suffers from very poor performance. This is likely to be due to the effects of interactions between the circuit components that have not been dealt with in the spacing of parameters in the orthogonal array. The predictive equations on which the optimisation through ANOM is based does not hold true for when such strong interactions exist.

However, the approach that utilises the least significant factor to tune the solution to give the best performance does allow some flexibility in the optimisation process and the best predicted solution does not have such poor performance. The following figures plot the response of the circuit for the low, medium and high noise conditions. 


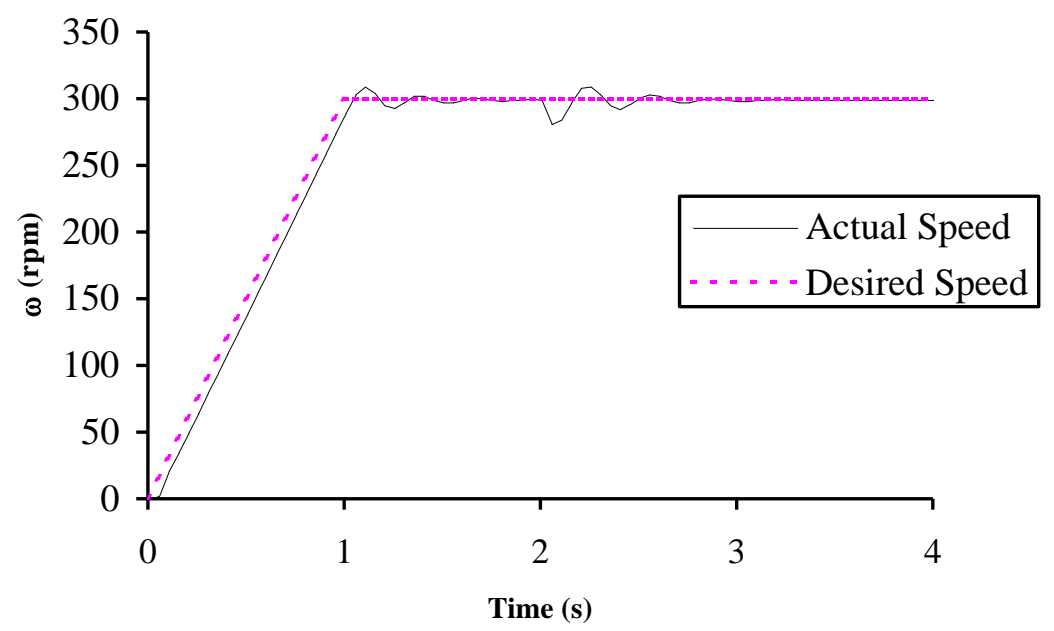

Figure 8. Response of predicted solution for noise factors at low values

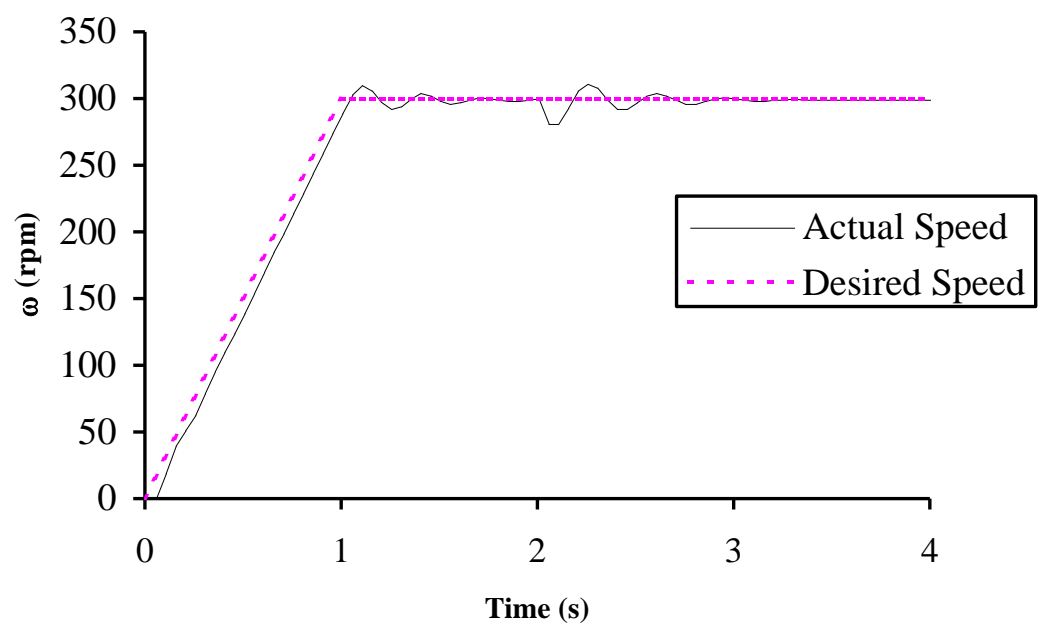

Figure 9. Response of predicted solution for noise factors at medium values

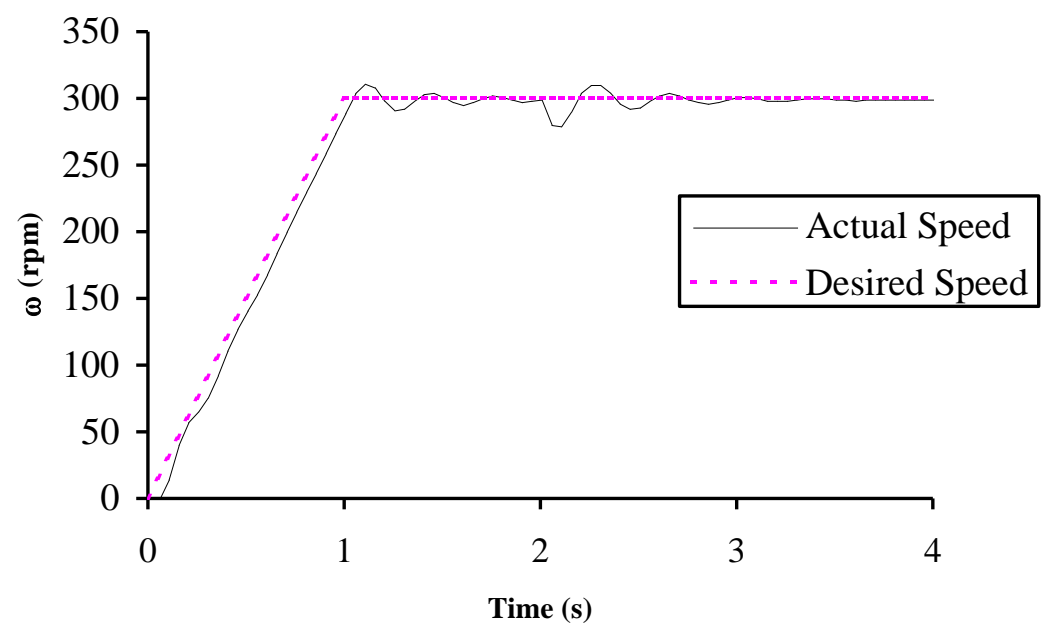

Figure 10. Response of predicted solution for noise factors at high values 
These graphs show that the performance of the best predicted solution differs from that of the best solution in the orthogonal array. The character of the speed response is more consistent across the three noise conditions with only minor instabilities in the initial ramp up to operating speed. In the best of the solutions found in the orthogonal array the instabilities in this region were very high, probably due to an overtuned controller.

However, the best of the predicted solutions does not maintain constant speed after the initial ramp period and the reaction to the change in load torque takes a considerable length of time to settle down. This possible indicates that the controller is undertuned.

\section{Discussion}

By considering the responses of both the best solution in the orthogonal array and the best predicted solution it can be seen that applying Taguchi methods blindly in the parameter sizing stage of fluid power circuit design is unlikely to lead to the design of high quality, robust circuits. One of the main reasons the approach cannot be applied blindly is due to the effects of interactions in the fluid power circuit that restrict the applicability of the predicted equations to find truly optimal and robust designs.

Whilst the effects of interactions are detrimental to the performance of the method there are other possible reasons why the approach has not resulted in satisfactory designs. Firstly, previous work on this circuit using a numerical optimisation algorithm [5] has shown that it is not easy to automatically select parameter values for a single operating condition let alone multiple operating conditions that represent faults in the circuit. The controller in the circuit does provide the circuit with the functional capacity to maintain constant speed as the load torque changes but it is likely that the tuning of the controller gains does not guarantee stability on a wide number of different operating conditions.

One other possible contributing factor relates to the use of dynamic simulation to assess the performance of each circuit. Previous work [6] has shown that the specification of a fixed time period at which the evaluation takes place can lead to an apparently acceptable performance when considering the numerical objective function value. However, these circuits are generally simulated using a variable time step integrator and plots of the performance parameters show that low errors exist at the specified times but instabilities exist between the evaluation points.

Other factors may have contributed to the poor results including the fact that the performance of individual solutions outside of the parameter optimisation experiment is assessed for noise conditions that are not present in the original optimisation. However, as the aim of this work is to design fault tolerant systems then there is some justification for this as it is difficult to determine which combination of circuit components may fail during operation. One other possibility is that modifying the standard array to include extra levels for each control factor has limited the extent to which the solutions in the array are representative of the entire solution space.

\section{Conclusions}

The results presented in this paper illustrate some of the problems in applying Taguchi methods to the parameter sizing stage of fluid power circuit design. The highly interactive nature of fluid power circuits implies that considerable thought is required in dealing with 
interactions when choosing and populating an appropriate orthogonal array for the parameter optimisation experiments.

The effects of interactions can be partially dealt with in a less rigorous approach by allowing parameters which are shown to have low significance on the performance of the solution to be changed so therefore acting as a buffer between highly interactive components.

Whilst some advances have been made towards designing fault tolerant systems using Taguchi methods the general conclusion is that given the interactive nature of fluid power circuits then the use of orthogonal arrays may not lead to acceptable solutions in all circumstances. However, there may be merit in embedding a signal to noise ratio calculation in an objective function used by a numerical optimisation algorithm so that robust systems can be design by a hybrid approach.

\section{References}

[1] TAGUCHI, G. (1989) Introduction to Quality Engineering (Asian Productivity Organisation).

[2] FOWLKES, W.Y. \& CREVELING, C.M. (1995) Engineering Methods for Robust Product Design (Addison-Wesley)

[3] SANCHEZ, S.M. (1994) A Robust Design Tutorial, Proceedings of the ASME Winter Simulation Conference, pp 106 - 113

[4] CONNOR, A.M. \& TILLEY, D.G. (1997) A Comparison of Two Methods Applied to the Optimisation of Fluid Power Circuits, Proceedings of the $10^{\text {th }}$ Bath International Fluid Power Workshop, pp12 - 27

[5] CONNOR, A.M. \& TILLEY, D.G. (1998) Optimisation of Power Transmission Systems Using a Discrete Tabu Search Method, Proceedings of the Bath Workshop on Power Transmissions and Motion Control, pp 153 - 164

[6] CONNOR, A.M. \& TILLEY, D.G. (1999) A Tabu Search Method for the Optimisation of Fluid Power Circuits, Proceedings of the IMechE Part I, Journal of Systems and Control Engineering, Vol 212, No 15, pp 373 - 381

[7] DEMIRCI, H.H. \& COULTER, J.P. (1996) A Comparative Study of Nonlinear Optimisation and Taguchi Methods Applied to the Intelligent Control of Manufacturing Processes, Journal of Intelligent Manufacturing, Vol 7, pp 23 - 28

[8] CHI, H-W. \& BLOEBAUM, C.C. (1995) Mixed Variable Optimisation Using Taguchi's Orthogonal Arrays, Proceedings of the ASME Design Engineering Technical Conference, pp $501-508$

[9] RAMAKRISHNAN, B. \& RAO, S.S. (1991) A Robust Optimisation Approach Using Taguchi's Loss Function for Solving Nonlinear Optimisation Problems, Proceedings of the ASME Advances in Design Automation Conference, pp 241 - 248

[10] JURAN, J.M. \& GRYNA, F.M. (1988) Juran's Quality Control Handbook (McGraw-Hill) 